\title{
MMP-9 and TIMP-1 in placenta of hypertensive disorder complicating pregnancy
}

\author{
YUYI ZHANG ${ }^{1 *}$, PENG LI ${ }^{2 *}$, YUE GUO $^{1}$, XIAOWAN LIU $^{3}$ and YUBO ZHANG ${ }^{4}$ \\ Departments of ${ }^{1}$ Gynecology and Obstetrics, and ${ }^{2}$ General Surgery, Xiangyang No. 1 People's Hospital, \\ Hubei University of Medicine, Xiangyang, Hubei 441000; ${ }^{3}$ Department of Gynecology, \\ People's Hospital of Xinjiang Uygur, Urumqi, Xinjiang Uygur Autonomous Region 830001; \\ ${ }^{4}$ Department of Stomatology, Quzhou No. 2 People's Hospital, Jinhua, Zhejiang 321000, P.R. China
}

Received November 1, 2018; Accepted April 19, 2019

DOI: $10.3892 / \mathrm{etm} .2019 .7591$

\begin{abstract}
Expression and characteristics of matrix metalloproteinase-9 (MMP-9) and TIMP metallopeptidase inhibitor-1 (TIMP-1) in the placenta of pregnancy induced hypertension (PIH) were detected in Uygur to analyze its correlation with $\mathrm{PIH}$, and to provide a theoretical basis for clinical work. Ninety cases of placental tissue patients who were hospitalized in the Department of Obstetrics of People's Hospital of Xinjiang Uygur from December 2014 to September 2016 were collected, including 30 cases of severe preeclampsia, 30 cases of mild preeclampsia, and 30 cases of normal group. The distribution of MMP-9 and TIMP-1 in placenta was mainly in the cytoplasm of trophoblast cells, vascular endothelial cells and villous mesenchymal cells. The distribution of MMP-9 positive particles in the placenta tissue of the severe group was significantly reduced. The difference of MMP-9 in the three groups was statistically significant. Severe group was statistically significantly different from normal and mild group. With the aggravation of PIH, positive expression of MMP-9 was gradually decreased. TIMP-1 was expressed in each group, and difference was not statistically significant. Positive expression ratio of MMP-9/TIMP-1 in severe group was lower than that in normal pregnancy and mild group, and positive expression ratio of the two groups became smaller as the condition worsened. Positive expression of MMP-9 in placental tissue of patients with PIH decreased significantly with the severity of PIH. TIMP-1 in placental tissue of PIH patients did not change much in each group, and had no significant correlation with PIH.
\end{abstract}

Correspondence to: Dr Yubo Zhang, Department of Stomatology, Quzhou No. 2 People's Hospital, 338 Xin'an Road, Jinhua, Zhejiang 321000, P.R. China

E-mail: b584xg@163.com

*Contributed equally.

Key words: TIMP metallopeptidase inhibitor-1, hypertensive disorders of pregnancy, matrix metalloproteinase-9, placenta, Uygur women

\section{Introduction}

Hypertensive disorders of pregnancy are unique. Hypertension, proteinuria, and edema are the main clinical symptoms that disappear after delivery. It is the second cause of maternal death (1). The incidence rate is $9.4-10.4 \%$ in China (2), and $7-12 \%$ worldwide (3). The basic pathophysiological changes are systemic small arterial spasm, endothelial damage and ischemia, and blood flow perfusion in various organs and systems of the whole body is reduced, leading to multiple organ damage (4). Its aetiology remains unclear (5). For severely ill patients, only the termination of pregnancy can prevent the condition from worsening. The incidence of latent preterm birth is increased (6). Recent studies have found that the occurrence of PIH is associated with dystrophic cell invasion into the uterus (7). The most prominent manifestation is abnormal changes in the hemodynamics of the placenta bed. It is characterized by large uterine spiral arterial resistance, narrow tube diameter, and greatly reduced blood perfusion (8). The degradation of extracellular matrix is the rate-limiting step of nourishing cell erosion. Matrix metalloproteinase-9 (MMP-9) is the only effective hydrolase secreted by trophoblast cells that can digest extracellular matrix (ECM). This study detected the expression and characteristics activity of MMP-9 and TIMP metallopeptidase inhibitor-1 (TIMP-1) PIH placenta, in Uygur to analyze its correlation with $\mathrm{PIH}$, with the aim to provide theoretical basis for clinical work.

\section{Patients and methods}

Collection of materials. Ninety cases of placental tissue specimens of Uygur patients who were hospitalized from December 2014 to September 2016 in the Department of Obstetrics of People's Hospital of Xinjiang Uygur (Urumqi, China). Clinical diagnosis was 30 cases of severe preeclampsia (severe group), and the maternal age was 22-35 years, with an average of 30.9 \pm 4.5 years, 36.1 (32.3-38.5) weeks of gestational age; 30 cases with mild preeclampsia (mild group), 22-35 years, average 29.0 4.0 years, 38.5 (37.9-39.4) weeks of gestational age; 30 cases of normal pregnancy, no medical and obstetric complications were the normal group, maternal gestational age 39.0 (38.2-40.2) weeks, age 22 to 35 years, average $29.7 \pm 4.0$ years. There was no significant difference in 
Table I. Expression of MMP-9 in placental tissues in each group $(n=30)$.

\begin{tabular}{lrrrr}
\hline & \multicolumn{4}{c}{ Grading } \\
\cline { 2 - 5 } Groups & - & + & ++ & +++ \\
\hline Normal & 3 & 10 & 8 & 9 \\
Mild & 3 & 2 & 14 & 11 \\
Severe & 6 & 18 & 5 & 1 \\
\hline
\end{tabular}

$\chi^{2}=25.28, \mathrm{P}<0.001$. MMP-9, matrix metalloproteinase-9.

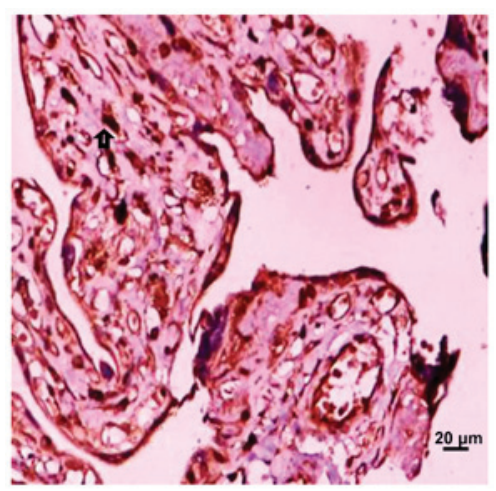

Figure 1. MMP-9 expressed in normal placental tissue (40x10). Arrow refers to positive particles. MMP-9, matrix metalloproteinase-9.

the number of births $(\mathrm{P}>0.05)$. None of the patients received blood transfusion or immunotherapy, and were excluded from chronic nephritis, essential hypertension, epilepsy, cerebral hemorrhage, diabetic ketoacidosis, hyperosmolar coma, hypoglycemia coma, malignant tumors and other internal surgical diseases. Inclusion criteria: i) According to the 'Obstetrics and Gynecology' diagnostic criteria for early severe preeclampsia, all patients were women with initial pregnancy, ii) signed an informed consent, and iii) with the permission of the Ethics Committee of the hospital, no drug treatment was received.

This study was approved by the Ethics Committee of People's Hospital of Xinjiang Uygur. Patients who participated in this research had complete clinical data. The signed informed consents were obtained from the patients or the guardians.

Specimen preparation. After the placenta was delivered, the placenta tissue of $2 \mathrm{~cm} \times 2 \mathrm{~cm} \times 0.5 \mathrm{~cm}$ was taken from the maternal surface to the fetal surface in the central place of the placenta, and fixed in $10 \%$ neutral formalin for $24 \mathrm{~h}$ at $22^{\circ} \mathrm{C}$, embedded in low temperature paraffin. The thicknes of sections was $4 \mu \mathrm{m}$. The microscope slides were washed and dried, and then immersed in a polylysine solution diluted in deionized water $(1: 10)$ for $5 \mathrm{~min}$, and baked in a $600^{\circ} \mathrm{C}$ oven for $1 \mathrm{~h}$ for anti-stripping treatment. In $1 \mathrm{ml}$ of reagent 2 (DAB substrate solution), 1 drop (about 50 microliters) of reagent 1 (DAB concentrate) was added and mixed, then DAB solution was made. Dip 3 times in PBS, 5 min every time at room temperature. The expression levels of MMP-9 and TIMP-1 were determined by immunohistochemical

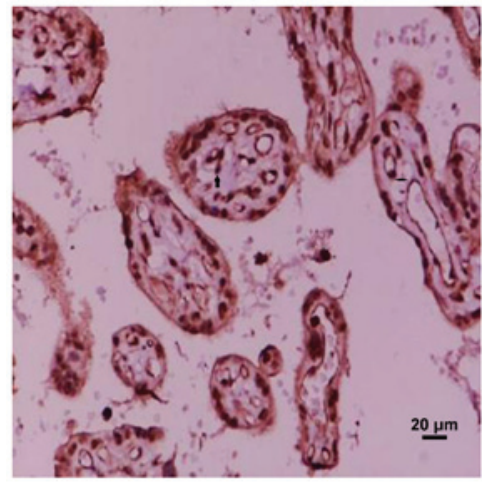

Figure 2. MMP-9 expressed in preeclampsia mild placental tissues. Arrow refers to positive particles. MMP-9, matrix metalloproteinase-9.

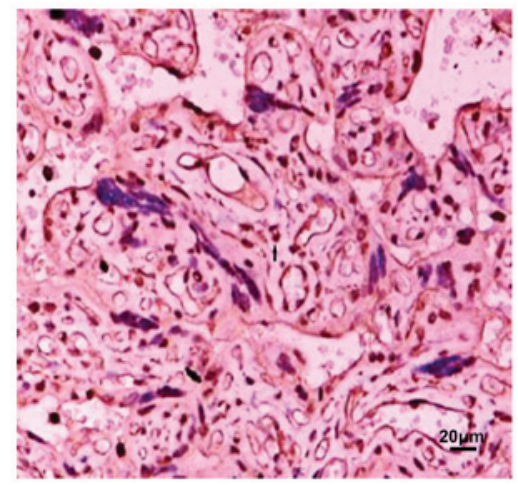

Figure 3. MMP-9 expressed in severe preeclampsia placental tissues. Arrow refers to positive particles. MMP-9, matrix metalloproteinase-9.

staining. Immunohistochemical antibodies were provided by ZS-BIO (Beijing, China), MMP-9 (cat. no. ZA-0562; dil 1:100) and TIMP-1 (cat. no. ZA-0429; dil 1:200) and performed strictly in accordance with the instructions. The microscope OLYMPUS-BX51 (Olympus Corp., Tokyo, Japan) was used.

Evaluating the dyeing results. Under the microscope, the cytoplasm showed brown-yellow granular or flaky staining as positive cells, and the following evaluation methods were established according to the cell staining intensity and coloring density. Five high power fields were counted and at least 1,000 cells were evaluated per slice. Coloring intensity: light coloring 1 point, moderate coloring 2 points, and strong coloring 3 points. Positive cells $<10 \% 1$ point, $10-49 \%$ 2 points, $50-79 \% 3$ points, and $\geq 80 \% 4$ points. The two items were multiplied to obtain the total score of each case stained. One to 3 were negative (-), 4 to 6 were weakly positive (+), 7 to 9 were positive $(++)$, and 10 to 12 were strongly positive $(+++)$.

Statistical analysis. SPSS 13.0 (SPSS, Inc., Chicago, IL, USA) was used to analyze the data, and the measurement data are represented by (mean $\pm \mathrm{SD}$ ). The Chi-square tests or Fisher's exact probability method was used for enumeration data.

\section{Results}

Localization and positive distribution of MMP-9 in placental tissue. MMP-9 is mainly distributed in the cytoplasm of 
Table II. Expression of TIMP-1 in placental tissues in each group $(\mathrm{n}=30)$.

\begin{tabular}{lrrrr}
\hline & \multicolumn{4}{c}{ Grading } \\
\cline { 2 - 5 } Groups & - & + & ++ & +++ \\
\hline Normal & 0 & 10 & 16 & 4 \\
Mild & 0 & 6 & 12 & 12 \\
Severe & 0 & 8 & 13 & 9
\end{tabular}

$\chi^{2}=0.817, \mathrm{P}>0.05$. TIMP-1, TIMP metallopeptidase inhibitor-1.

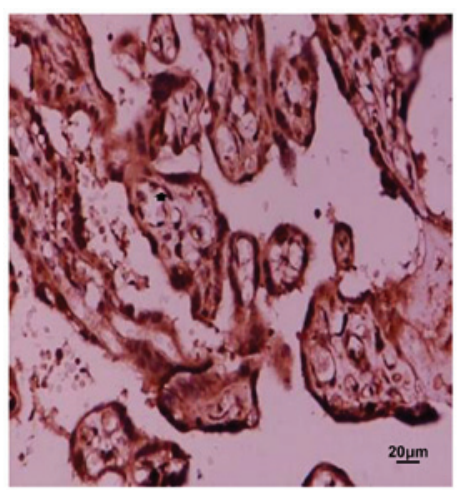

Figure 4. Expression of TIMP-1 in normal placental tissue. Arrow refers to positive particles. MMP-9, matrix metalloproteinase-9.

trophoblast cells, vascular endothelial cells and villous mesenchymal cells, but the expression intensity is different in each group. The location of MMP-9 in the placenta tissue of the preeclampsia group was the same as that in the normal late pregnancy group. Among the four groups of placental grade $(-),(+),(++),(+++)$, the difference in the number of people in normal, mild and severe group was statistically significant $\left(\chi^{2}=25.28, \mathrm{P}<0.001\right)$. In the placenta grade $(-)$, the maximum number of people in the severe group was 6 ; in the placenta grade (+). Most of the positive group was concentrated in the severe group, and the least positive group was in the mild group; the placenta grade $(++)$. Most positive patients were concentrated in mild group, and least number of positive patients were in severe group. Number of positive patients with placenta grade $(+++)$ was concentrated in mild group, and least positive group was the severe group. Distribution of MMP-9 positive particles in the placenta tissue of severe preeclampsia group was significantly reduced (Figs. 1-3 and Table I). Pairwise comparison was carried out between mild and severe, mild and normal, severe and normal groups. There was no significant difference between mild and normal group. The difference in MMP-9 between the other two groups was statistically significant, that is, MMP-9 was expressed differently in the three groups. With the aggravation of PIH, the distribution of positive particles of MMP-9 was gradually reduced.

Location and positive expression of TIMP-1 in placenta tissue. TIMP-1 is mainly distributed in the cytoplasm of trophoblast

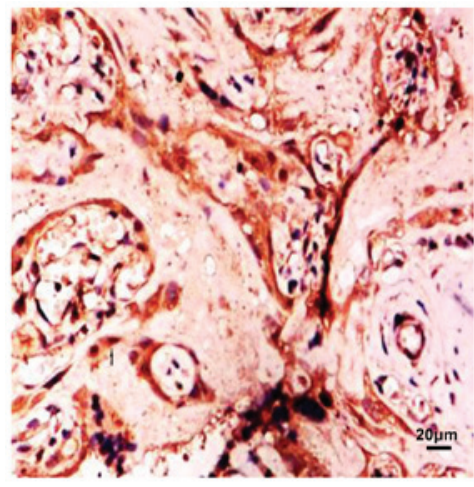

Figure 5. Expression of TIMP-1 in mild placental tissue of preeclampsia Arrow refers to positive particles. TIMP-1, TIMP metallopeptidase inhibitor-1.

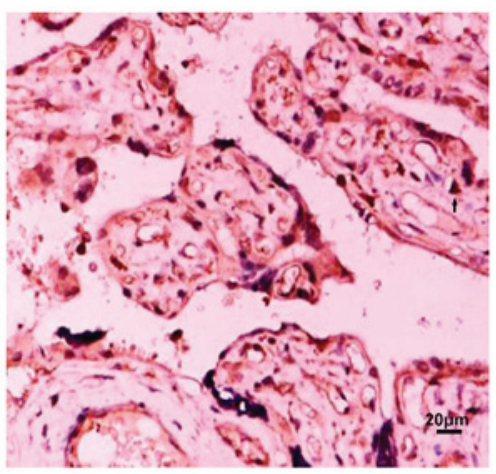

Figure 6. Expression of TIMP-1 in severe preeclampsia placenta $(40 \times 10)$ Arrow refers to positive particles. TIMP-1, TIMP metallopeptidase inhibitor-1.

cells, vascular endothelial cells and villous mesenchymal cells. Pairwise comparison was carried out between mild and severe, mild and normal, severe and normal groups. There was no significant difference in the four groups of placental grades $(-),(+),(++)$, and $(+++)\left(\chi^{2}=0.817, \mathrm{P}>0.05\right)$, number of positive in normal, mild and severe group was 0 in placenta grade (-); most of the positive in the placenta grade $(+)$, concentrated in normal group, the least number of positives were in mild group, the highest number of positive placenta (++) was concentrated in normal group. The positive number of placenta in the $(+++)$ group was concentrated in mild group, and least positive group was normal group. Number of positives in the three groups is basically balanced (Figs. 4-6, Table II).

Positive expression ratio of MMP-9, TIMP-1, MMP-9/TIMP-1 in placenta in each group. MMP-9 was expressed differently in the three groups of placental tissues. With the aggravation of PIH, the distribution of positive particles of MMP-9 was gradually reduced. MMP-9 positive particles in the placenta tissue of the severe preeclampsia group were significantly reduced. TIMP-1 was expressed in each group, and the difference was not statistically significant. The positive expression ratio of MMP-9/TIMP-1 in the severe group was lower than that in the normal pregnancy group and the mild group, and the positive expression ratio of the two groups gradually decreased as the condition worsened. 


\section{Discussion}

Matrix metalloproteinases (MMPs) are a class of proteolytic enzymes with similar structures and complex functions. It is the most important enzyme in matrix degradation. It is named after $\mathrm{Zn}^{2+}$ and $\mathrm{Ca}^{2+}$ and belongs to the family of $\mathrm{Zn}^{2+}$-dependent enzymes. The interaction of MMPs with the basement membrane (BM) is an important starting signal for tumor invasion and metastasis (9). MMP-9 is the only effective hydrolase secreted by trophoblast cells that can digest ECM. It has been reported to be involved in extracellular matrix (ECM) remodeling and processes of placental angiogenesis with structural spiral arteries transformation, which precedes proper trophoblastic invasion (10). TIMP-1 is a tissue inhibitory factor corresponding to MMP-9 and is normally secreted by the same kind of cells that secrete MMP-9. Usually combined with the MMP-9 zymogen form into a 1:1 complex, which is linked by non-covalent bonds, thereby preventing the activation of the zymogen and inhibiting the activity of MMP-9 to degrade collagen. Even when activated MMP-9 acts on the basement membrane, it depends on the balance of MMP-9 and its inhibitory factor (TIMP-1), only when the balance is broken (i.e. 30 MMP-9/TIMP-1 at $>1: 1$ ), biological activity is produced (11). Therefore, the balanced expression of MMP-9 and TIMP-1 has profound implications for regulating the depth of trophoblast invasion into the uterus.

Previous studies on serum MMP-9 and TIMP-1 in patients with pregnancy-induced hypertension showed that serum MMP-9 levels in severe group were significantly lower than those in normal group and mild group $(\mathrm{P}<0.05)$, but there was no significant difference between mild group and control group $(\mathrm{P}>0.05)$, without statistical significance. There was no significant difference in serum TIMP-1 between severe, mild and normal groups ( $\mathrm{P}>0.05)$, and the concentration of TIMP-1 in the three groups did not change much (12), which is consistent with this study.

Laird (13) found that the placenta implantation process is strictly limited by the time and space of MMPs and TIMPs, thus controlling the depth of trophoblastic infiltration. The physiological changes in most patients with PIH are limited to the aponeurotic segment of the uterine artery. This study found that MMP-9 in placenta tissue is mainly distributed in the cytoplasm of trophoblast cells, vascular endothelial cells and villous mesenchymal cells, but the expression intensity is different in each group, and MMP-9 is positive as the severity of PIH is aggravated. The expression of the particles is gradually decreasing. TIMP-1 and MMP-9 were consistently distributed in each group, and the difference was not statistically significant. The positive expression of MMP-9/TIMP-1 in the severe group was lower than that in the normal pregnancy group and the mild group, and the disease was aggravated. The positive expression ratio of the two gradually decreased. It was observed that the expression of MMP-9 decreased in the severe group, but the specific inhibitor TIMP-1 had no corresponding change, and the equilibrium state between the two changed. The expression of MMP-9 and TIMP-1 is related to the occurrence of preeclampsia and the development of the disease. The level of MMP-9 in the placental tissue decreased, and the balance between MMP-9 and TIMP- 1 changed. The erosive cell function was affected, which can lead to shallow implantation of the placenta, resulting in ischemia and hypoxia of the placenta, which eventually leads to maternal systemic vascular endothelial damage, thus showing the clinical symptoms of PIH. Recent studies have also shown that the extent of injury or disease can be estimated by measuring MMP-9 concentration, and the prognostic effect of treatment can be preliminarily predicted (7).

Moreover, in vitro experiments have shown that the trophoblast infiltration ability is significantly reduced because the expression of MMP-9 is lower than the normal level required for infiltration (8). The above suggests that MMP-9 and TIMP-1 act as a pair of mutually restrictive factors playing an important role in the pathogenesis of PIH.

\section{Acknowledgements}

Not applicable.

\section{Funding}

No funding was received.

\section{Availability of data and materials}

The datasets used and/or analyzed during the current study are available from the corresponding author on reasonable request.

\section{Authors' contributions}

YuyiZ wrote the manuscript. YuyiZ and YuboZ were responsible for immunohistochemical staining. PL and YG evaluated the results of dyeing. XL and YuyiZ worked on specimen preparation. All the authors read and approved the final manuscript.

\section{Ethics approval and consent to participate}

This study was approved by the Ethics Committee of People's Hospital of Xinjiang Uygur (Urumqi, China). Patients who participated in this research had complete clinical data. The signed informed consents were obtained from the patients or the guardians.

\section{Patient consent for publication}

Not applicable.

\section{Competing interests}

The authors declare that they have no competing interests.

\section{References}

1. Cerdeira AS and Karumanchi SA: Angiogenic factors in preeclampsia and related disorders. Cold Spring Harb Perspect Med 2: 006585, 2012.

2. Vadhera RB and Simon M: Hypertensive emergencies in pregnancy. Clin Obstet Gynec 57: 797-805, 2014.

3. Winn VD, Gormley M and Fisher SJ: The impact of preeclampsia on gene expression at the maternal-fetal interface. Pregnancy Hypertens 1: 100-108, 2011. 
4. Kleinrouweler CE, Wiegerinck MM, Ris-Stalpers C, Bossuyt PM van der Post JA, von Dadelszen P, Mol BW and Pajkrt E; EBM CONNECT Collaboration: Accuracy of circulating placental growth factor, vascular endothelial growth factor, soluble fms-like tyrosine kinase 1 and soluble endoglin in the prediction of pre-eclampsia: A systematic review and meta-analysis. BJOG 119: 778-787, 2012.

5. Chen J, Yu YH, Wang ZJ, Qin W and Zhang Q: Relationship between preeclampsia umbilical blood flow and perinatal outcomes. Nan Fang Yi Ke Da Xue Xue Bao 29: 745-746, 2009 (In Chinese)

6. Zhu J, Zhong M, Pang Z and Yu Y: Dysregulated expression of matrix metalloproteinases and their inhibitors may participate in the pathogenesis of pre-eclampsia and fetal growth restriction. Early Hum Dev 90: 657-664, 2014.

7. Shi H, Liu L, Liu LM, Geng J and Chen L: Inhibition of tumor growth by $\beta$-elemene through downregulation of the expression of uPA, uPAR, MMP-2, and MMP-9 in a murine intraocular melanoma model. Melanoma Res 25: 15-21, 2015.

8. McCool WF, Durain D and Davis M: Overview of latest evidence on uterine fibroids. Nurs Womens Health 18: 314-332, 2014

9. Jaiswar SP, Gupta A, Sanchan R, Natu SN and Shaili M: Latic dehydrogenase: A biochemical marker for preeclampsiaeclampsia. J Obstet Gynaecol India 61: 645-648, 2011.
10. Maged AM, Aid G, Bassiouny N, Eldin DS, Dahab S and Ghamry NK: Association of biochemical markers with the severity of pre-eclampsia. Int J Gynaecol Obstet 136: 138-144, 2017.

11. Sun C, Zhang Q, Hu B and Zhang K: Investigation of the association between matrix metalloproteinase-9 genetic polymorphisms and development of pre-eclampsia in Chinese pregnant women. Genet Mol Res 15: 1-6, 2016.

12. Zhang YY, Ren HY, Wang YT, Deng J, Liu XW and Li XY: Expression of MMP-9 and TIMP-1 in placental tissue of pregnancy-induced hypertension in Uigur women in Xinjiang. Progress Modern Biomed 19: 3710-3712, 2009 (In Chinese).

13. Laird SM: Metalloproteinases and tissue inhibitor ormetalloproteinase-1 (TIMP-1) in endometrial flushings from pre-and post-menopausal women and from women with endometrial adenocarcinoma. J Reprod Fertil 115: 225-232, 1999.

This work is licensed under a Creative Commons Attribution-NonCommercial-NoDerivatives 4.0 International (CC BY-NC-ND 4.0) License. 